\title{
Upper lip metastasis of sarcomatoid carcinoma of the lung - an unusual site of disease: a case report
}

\author{
Tri Le ${ }^{1}$, Melissa Mayer ${ }^{2}$, Joseph Sailors ${ }^{3}$, David E. Gerber ${ }^{1,2^{*}}$ and John M. Truelson ${ }^{2,4}$
}

\begin{abstract}
Background: Lip metastases are rare clinical events that are frequently mistaken for other diagnoses. For sarcomatoid lung carcinoma, a rare histologic variant of non-small cell lung cancer, the incidence and pattern of cutaneous spread is poorly understood.

Case presentation: We present a case of a 79-year-old African American man with a rapidly progressive upper lip cutaneous lesion that provided the first evidence of distant metastatic spread of sarcomatoid lung carcinoma.

Conclusions: This is the first reported case of lip metastasis in sarcomatoid lung carcinoma. It highlights the importance of maintaining a high level of suspicion for metastatic disease in the presence of new cutaneous findings as they may be the first evidence of advanced disease.
\end{abstract}

Keywords: Lip, Cutaneous, Metastasis, Lung cancer, Sarcomatoid

\section{Background}

Sarcomatoid lung carcinoma is an aggressive and rare histologic variant of non-small cell lung cancer (NSCLC; 0.3 to $1.3 \%$ of all lung malignancies) that is morphologically defined by components of sarcoma or sarcoma-like differentiation (for example, spindles and/or giant cells) [1]. Prompt recognition of metastatic progression is critical to management, as staging determines treatment and prognosis. The skin provides a noninvasive opportunity to identify metastatic disease, but the pattern and incidence of cutaneous spread in this disease remains unknown. Here, we present a case of rapidly progressive upper lip cutaneous metastasis from a case of sarcomatoid lung carcinoma. To the best of our knowledge, it is the first documented case of lip metastasis in this disease, establishing the lip as a potential site of spread.

\footnotetext{
* Correspondence: david.gerber@utsouthwestern.edu

${ }^{1}$ Department of Internal Medicine, University of Texas Southwestern Medical Center, 5323 Harry Hines Blvd., Dallas, TX 75390, USA

${ }^{2}$ Harold C. Simmons Cancer Center, University of Texas Southwestern Medical Center, 2201 Inwood Road, 3rd Floor, Suite 500, Dallas, TX 75390-9125, USA

Full list of author information is available at the end of the article
}

\section{Case presentation}

A 79-year-old African American man with a history notable for heavy tobacco use, traumatic brain injury following a motor vehicle accident, hypothyroidism, and chronic lymphocytic leukemia last requiring therapy 4 years previously presented with 1 month of persistent cough. On a chest X-ray, a large mass in the mid-lung zone of his left lung was noted. Chest computed tomography $(\mathrm{CT})$ demonstrated a mass in the upper lobe of his left lung measuring $7.7 \times 8.7 \mathrm{~cm}$ and a $1.1 \mathrm{~cm}$ left hilar lymph node (Fig. 1a). A CT-guided percutaneous biopsy of the lung mass revealed malignant spindled and focally epithelioid neoplasm with extensive necrosis with poor differentiation favoring sarcomatoid carcinoma (Fig. 1b). Immunohistochemistry demonstrated positive staining for epithelial markers CAM5.2 (Fig. 1c), cytokeratin (CK) 5/6, CK903, and CK7, and negative staining for thyroid transcription factor 1 (TTF-1), p63, desmin, S100, and paired-box gene 8 (PAX8).

At this time, he was also noted to have an ulcerated area on his upper lip on which topical anesthetic agents were placed. Seven days later, a follow-up examination revealed painful and substantial upper lip swelling. An abscess was suspected, systemic antibiotics were prescribed, and he was referred urgently to our Otolaryngology 


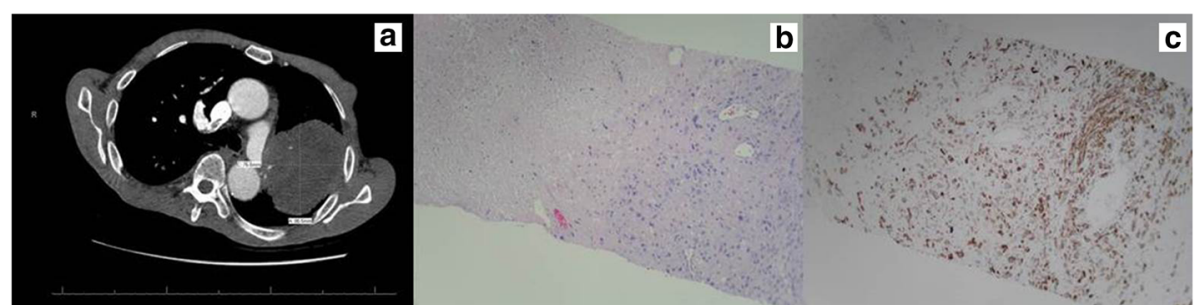

Fig. 1 Primary sarcomatoid non-small cell lung cancer. a A $7.7 \times 8.7 \mathrm{~cm}$ mass in the upper lobe of the left lung and the lingula causing extrinsic compression of the left pulmonary artery and its branches with extension into the left superior pulmonary vein, mediastinum, and probably the pericardium. b Core fragments of lung mass demonstrating spindle cell processes, large areas of necrosis, and intermediate-sized nuclei (100x). c Immunohistochemical staining with cells positive for CAM5.2 (100X)

service. He was found to have a $2.7 \mathrm{~cm}$ upper lip mass with normal overlying skin. A CT of his neck revealed a $2.1 \mathrm{~cm}$ anteroposterior $(\mathrm{AP}) \times 3.6 \mathrm{~cm}$ transverse $\times 3.8 \mathrm{~cm}$ craniocaudal (CC) right upper lip mass with surrounding inflammatory change (Fig. 2a). An incisional biopsy demonstrated metastatic sarcomatoid carcinoma, with morphologic and immunohistochemical characteristics similar to the primary lung lesion (Fig. 2b, c).

Subsequent imaging demonstrated two intracranial metastases. He received palliative radiation 3000 cGy in 10 fractions to his upper lip and 3000 cGy in 10 fractions to his brain. He continued to decline functionally; he was admitted to our in-patient hospice unit, and died 39 days after initial diagnosis of the primary sarcomatoid lung cancer.

\section{Discussion}

Sarcomatoid lung carcinoma is an aggressive and rare histologic variant of NSCLC ( 0.3 to $1.3 \%$ of all lung malignancies) that is morphologically defined by components of sarcoma or sarcoma-like differentiation (for example, spindles and/or giant cells) [1]. There are five primary subgroups recognized under the 2004 and the 2015 World Health Organization Classification of Tumors of the Lung: pleomorphic carcinoma, spindle cell carcinoma, giant cell carcinoma, carcinosarcoma, and pulmonary blastoma [1-4]. Relative to other NSCLC histologies, sarcomatoid carcinoma has poorer stage-by-stage prognosis [3] and earlier recurrence after resection. The average age at diagnosis is 60 to 65 years [1,3,5-15]. Several studies suggested an increased male prevalence $[1,2,4,5,8,11]$, while others, including the largest epidemiologic study to date using the Surveillance, Epidemiology, and End Results database, suggested a nearly 1-to-1 gender ratio $[3,12]$. Sarcomatoid carcinoma is associated with heavy tobacco smoking history $[1,9,15]$. Clinical symptoms are nonspecific (for example, cough, hemoptysis, dyspnea, thoracic pain, weight loss, fatigue, and fever due to recurrent pneumonia) and are often related to pulmonary tumor localization $[1,9,11,14]$.

In the limited experience reported for this aggressive disease, treatment varies significantly with stage. Local disease appears to have a good outcome with surgical resection alone [15]. In metastatic disease, systemic chemotherapy continues to be to be the mainstay of treatment. Unfortunately, the response to conventional NSCLC therapy is poorer than that of more common histologic variants [16]. Given the distinction in prognosis and treatment between local disease and distant spread, it is critical to identify metastatic disease early. Cutaneous involvement involving the face is a readily available, noninvasive opportunity for physicians to identify metastatic disease in the absence of sites of spread.

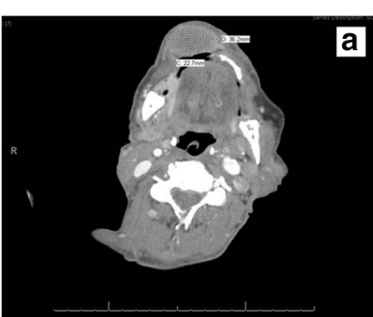

a

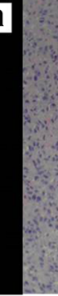

Fig. 2 Sarcomatoid non-small cell lung cancer metastasis to upper lip. a A $2.1 \mathrm{~cm}$ anteroposterior $\times 3.6 \mathrm{~cm}$ transverse $\times 3.8 \mathrm{~cm}$ craniocaudal rim-enhancing mass involving the right upper lip with mild surrounding inflammatory changes. b Scant benign superficial epithelium with an overriding subepithelial spindle and epithelioid cell neoplastic population with intermediate-sized nuclei with some degeneration and moderate amounts of pink cytoplasm, scattered multinucleated giant cells, and small intratumoral foci of necrosis (100X). c Immunohistochemical staining with scattered MNF116-positive cells (100x) 
Malignancy involving the lip is a rare clinical event and largely represented by primary squamous cell (49\%) and basal cell (40\%) carcinomas. Other etiologies include salivary gland origin (9\%) and metastatic cancer to the lip (2\%) [17]. Among the few reported cases of lip metastases in the literature, lung, gastric adenocarcinoma, breast, lymphoma, and renal cell carcinoma are described [18]. Clinical manifestation is often a submucosal mass with intact overlying skin, occasionally with ulceration, as was observed in this case [19]. To the best of our knowledge, this is the first reported case of sarcomatoid carcinoma of the lung metastasizing to the lip. High suspicion for metastatic disease should be maintained when new skin findings are observed in patients with a known cancer diagnosis, with low threshold for early histologic confirmation by biopsy.

\section{Conclusions}

This case establishes the lip as a potential site of metastatic spread for sarcomatoid lung carcinoma. Lip and other cutaneous metastases provide an easily identifiable opportunity to diagnose distant disease in patients with cancer diagnoses.

\section{Abbreviations}

CK: Cytokeratin; CT: Computed tomography; NSCLC: Non-small cell lung cancer

\section{Acknowledgements}

Not applicable.

Funding

None.

\section{Authors' contributions}

TL acquired data, drafted, and revised the manuscript. MM acquired data for the manuscript. JS acquired data and revised the manuscript. DG was involved in conception and revised the manuscript. JT acquired data and revised the manuscript. All authors read and approved the final manuscript.

\section{Competing interests}

The authors declare that they have no competing interests.

\section{Consent for publication}

Written informed consent was obtained from the patient for publication of this Case Report and any accompanying images. A copy of the written consent is available for review by the Editor-in-Chief of this journal.

\section{Ethics approval and consent to participate}

Not applicable.

\author{
Author details \\ ${ }^{1}$ Department of Internal Medicine, University of Texas Southwestern Medical \\ Center, 5323 Harry Hines Blvd., Dallas, TX 75390, USA. ${ }^{2}$ Harold C. Simmons \\ Cancer Center, University of Texas Southwestern Medical Center, 2201 \\ Inwood Road, 3rd Floor, Suite 500, Dallas, TX 75390-9125, USA. ${ }^{3}$ Department \\ of Pathology, University of Texas Southwestern Medical Center, 5323 Harry \\ Hines Blvd., Dallas, TX 75390, USA. ${ }^{4}$ Department of Otolaryngology, University \\ of Texas Southwestern Medical Center, 5323 Harry Hines Blvd., Dallas, TX \\ 75390, USA.
}

Received: 7 September 2016 Accepted: 13 December 2016

Published online: 19 January 2017

\section{References}

1. Travis WD, Brambilla E, Müller-Hermelink HK, Harris CC. World Health Organization Classification of Tumours. Pathology and genetics of tumors of the lung, pleura, thymus, and heart. Lyon: IARC Press; 2004. p. 53-8.

2. Travis WD, Brambilla E, Nicholson AG, et al. The 2015 World Health Organization Classification of Lung Tumors: Impact of Genetic, Clinical and Radiologic Advances Since the 2004 Classification. J Thorac Oncol. 2015;10(9):1243-60.

3. Martin LW, Correa AM, Ordonez NG, et al. Sarcomatoid carcinoma of the lung: a predictor of poor prognosis. Ann Thorac Surg. 2007:84(3):973-80.

4. Venissac N, Pop D, Lassalle S, Berthier F, Hofman P, Mouroux J. Sarcomatoid lung cancer (spindle/giant cells): an aggressive disease? J Thorac Cardiovasc Surg. 2007;134(3):619-23.

5. Italiano A, Cortot $A B$, llie $M$, et al. EGFR and KRAS status of primary sarcomatoid carcinomas of the lung: implications for anti-EGFR treatment of a rare lung malignancy. Int J Cancer. 2009;125(10):2479-82.

6. Jiang $X$, Liu Y, Chen C, et al. The value of biomarkers in patients with sarcomatoid carcinoma of the lung: molecular analysis of 33 cases. Clin Lung Cancer. 2012;13(4):288-96.

7. Nishida K, Kobayashi Y, Ishikawa Y, et al. Sarcomatoid adenocarcinoma of the lung: clinicopathological, immunohistochemical and molecular analyses. Anticancer Res. 2002;22(6B):3477-83.

8. Nakajima M, Kasai T, Hashimoto H, Iwata Y, Manabe H. Sarcomatoid carcinoma of the lung: a clinicopathologic study of 37 cases. Cancer. 1999;86(4):608-16.

9. Chang YL, Lee YC, Shih JY, Wu CT. Pulmonary pleomorphic (spindle) cell carcinoma: peculiar clinicopathologic manifestations different from ordinary non-small cell carcinoma. Lung Cancer. 2001;34(1):91-7.

10. Park JS, Lee $Y$, Han J, et al. Clinicopathologic outcomes of curative resection for sarcomatoid carcinoma of the lung. Oncology. 2011;81(3-4):206-13.

11. Fishback NF, Travis WD, Moran CA, Guinee Jr DG, McCarthy WF, Koss MN. Pleomorphic (spindle/giant cell) carcinoma of the lung. A clinicopathologic correlation of 78 cases. Cancer. 1994;73(12):2936-45.

12. Yendamuri S, Caty L, Pine M, et al. Outcomes of sarcomatoid carcinoma of the lung: a Surveillance, Epidemiology, and End Results Database analysis. Surgery. 2012;152(3):397-402.

13. Rossi G, Cavazza A, Sturm N, et al. Pulmonary carcinomas with pleomorphic, sarcomatoid, or sarcomatous elements: a clinicopathologic and immunohistochemical study of 75 cases. Am J Surg Pathol. 2003;27(3):311-24.

14. Pelosi G, Fraggetta F, Nappi O, et al. Pleomorphic carcinomas of the lung show a selective distribution of gene products involved in cell differentiation, cell cycle control, tumor growth, and tumor cell motility: a clinicopathologic and immunohistochemical study of 31 cases. Am J Surg Pathol. 2003;27(9):1203-15.

15. Davis MP, Eagan RT, Weiland LH, Pairolero PC. Carcinosarcoma of the lung: Mayo Clinic experience and response to chemotherapy. Mayo Clin Proc. 1984;59(9):598-603.

16. Ouziane I, Boutayeb S, Mrabti H, Lalya I, Rimani M, Errihani H. Sarcomatoid carcinoma of the lung: a model of resistance of chemotherapy. N Am J Med Sci. 2014;6(7):342-5.

17. Czerninski R, Zini A, Sgan-Cohen HD. Lip cancer: incidence, trends, histology and survival: 1970-2006. Br J Dermatol. 2010;162(5):1103-9.

18. Jatti D, Puri G, Aravinda K, Dheer DS. An atypical metastasis of renal clear cell carcinoma to the upper lip: a case report. J Oral Maxillofac Surg. 2015;73(2):371. e1-6.

19. Hirshberg A, Shnaiderman-Shapiro A, Kaplan I, Berger R. Metastatic tumours to the oral cavity - pathogenesis and analysis of 673 cases. Oral Oncol. 2008;44(8):743-52. 